\title{
Mitochondrial myopathy, encephalopathy, lactic acidosis, and strokelike episodes with recurrent abdominal symptoms and coenzyme Q10 administration
}

\author{
MITSUTOSHI YAMAMOTO, ${ }^{*}$ TAKESHI SATO,$\dagger$ MIDORI ANNO,$\dagger$ \\ HIROSHI UJIKE, * MOTOHISA TAKEMOTO \\ From the Department of Neurology, Kagawa Central Hospital, Takamatsu, ${ }^{*}$ Department of Neurology, \\ Juntendou University School of Medicine, Tokyo, $\dagger$ and the Department of Neurosurgery, Sumitomo Beshi \\ Hospital, Niihama
}

SUMMARY A male with mitochondrial myopathy, encephalopathy, lactic acidemia, and strokelike episodes is reported. He had also recurrent episodes of ileus. Muscle biopsy revealed ragged-red fibres. The cytochemistry of cytochrome c oxidase (CCO) showed scattered nonstained fibres, while all muscle fibres were heavily stained by immunocytochemistry using $\mathrm{CCO}$ antibody. These findings suggest that partical CCO deficiency may be present in the skeletal muscles of the patient. NADH cytochrome $\mathrm{c}$ reductase in the patient's muscle mitochondria was low compared with normal controls (about 26\%), although succinate cytochrome c reductase was normal. Coenzyme Q10 administration $(90 \mathrm{mg} /$ day) did not improve CSF lactate levels, but did decrease plasma lactate levels. His muscle weakness slightly improved.

Mitochondrial myopathy denotes a group of various disorders with mitochondrial abnormalities. In 1977, Shapira et $_{\text {al }}{ }^{1}$ noted a group of diseases characterised by central nervous system (CNS) symptoms, and advocated the term mitochondrial encephalomyopathy, suggesting the presence of a common metabolic disorder in the muscle and CNS. Among patients with this disorder, Pavlakis et $a l^{2}$ described two of their own and nine in the literature showing mitochondrial myopathy, encephalopathy, lactic acidosis, and strokelike episodes (MELAS), and considered this condition to be a distinct clinical entity. We examined enzyme activity in the mitochondrial electron transport system in a patient with MELAS, and studied the effects of the administration of coenzyme Q10 (CoQ10), an important component of the system. Clinical features as well as the results of this treatment are reported.

Address for reprint requests: Dr M Yamamoto, Department of Neurology, Kagawa Central Hospital, 5-4-16 Bancho, Takamatsu 760, Japan.

Received 5 December 1986 and in revised form 8 April 1987. Accepted 8 May 1987

\section{Case report}

The patient was a 22 year old male without a relevant past or family history except that his mother had undergone an operation for meningioma. The patient showed normal development until 15 years of age. He presented with severe headache and vomiting on 18 May 1979, which continued for 2 hours, and vomiting accompanied by left hemianopsia on the next day. He was admitted to a local hospital. CT scans were normal on admission, but the headache contined for one week. Tremulous movements of extremities and trunk, and loss of consciousness were observed during hospitalisation. CT scan on 25 May revealed a low density area in the right occipital region (fig la), which had enlarged by 8 June (fig lb). A diagnosis of cerebral infarction of the right occipital lobe was made. Electroencephalograms (EEG) in March, 1980 showed paroxysmal high voltage slow waves but no spikes. As the patient developed a headache on the left side, right hemianopsia, and gait disturbance, he was admitted to Sumitomo Besshi Hospital on 9 April 1980. CT scans showed a low density area in the right occipital lobe on admission, and in the left occipital region 5 days after (fig lc). On the same day, small spike waves were noted in EEG, and treatment with phenytoin was initiated. Abdominal pain occurred on 23 April and laparotomy was performed due to ileus. However, no venous congestion was noted, and discolouration of the ileum became normal during the operation. With relief of the symptoms, he was dis- 

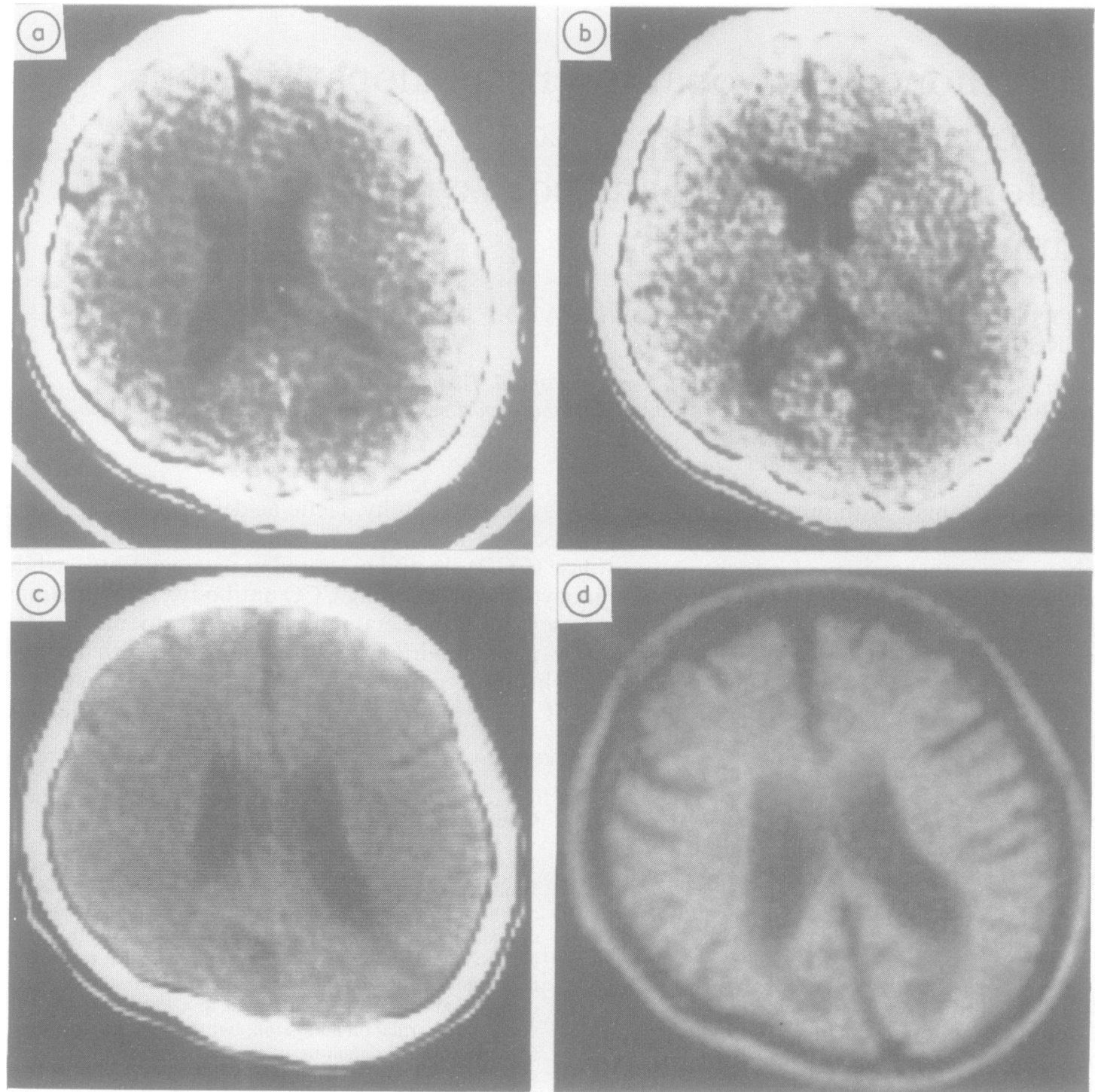

Fig 1 CT scan and MRI, (a) low density in the right occipital lobe (25 May 1979), (b) developed low density (8 June 1979), (c) low density area in the left occipital lobe (4 April 1980), (d) low density MRI (SE:320, 30) showed bilateral areas in occipital lobe (14 April 1985).

charged in June. The patient graduated from high school in 1982. In May, 1983, numbness and weakness of legs developed, and the patient was admitted. During this admission, three episodes of disorientation of 4-5 days duration were observed. Neurosensory hearing disturbance appeared in July, 1983. He was discharged in April, 1984 as the symptoms were moderately relieved. Complaining of headache and nausea on 9 May 1984, then vomiting and visual disturbance on the next day, the patient was readmitted. He subsequently exhibited unstable gait accompanied by severe headache and vomiting, but was discharged in July as the symptoms disappeared. He noted weakness of legs in February, 1985, and became unable to stand up 2 days later. On the next admission, phenytoin intoxication was noted. After recovery from the intoxication, the patient occasionally presented with myoclonic movements of the bilateral arms, and was admitted to Kagawa Central Hospital for examination in March, 1985. No clear abnormalities were noted 
in general haematological, biochemical, or cerebral fourvessel angiographic studies prior to the admission.

On admission, the patient was $160 \mathrm{~cm}$ tall and weighed $31.5 \mathrm{~kg}$. Neurological findings included bilateral tubular vision, severe hearing disturbance, moderate weakness and atrophy of the limb muscles, reduction of deep tendon reflexes, and dementia (WAIS 25 , partially due to hearing disturbance). External ocular movement, optic nerves, or the retina showed no abnormalities.

Blood analysis on admission showed normal protein, glucose, electrolytes, complete blood counts, erythrocyte sedimentation rate, BUN, phosphate, free fatty acid, vitamin B, ammonia, CK, GOT, GPT, and LDH levels. Blood gas analysis was normal. Thyroid and parathyroid functions were normal. Resting and fasting early morning levels of lactate and pyruvate were elevated to $4 \cdot 2 \mathrm{mmol} / 1$ (normal: $0.90 \pm 0.50 \mathrm{mmol} / \mathrm{l}, \mathrm{n}=20$, mean $\pm \mathrm{SD}$ ) and $135.2 \mu \mathrm{mol} / \mathrm{l}$ (normal: $45.4 \pm 22.7 \mu \mathrm{mol} / 1, \mathrm{n}=20$ ), respectively). A marked increase in lactate was noted in mild exercise tolerance tests.

No abnormalities were observed in lactate or pyruvate level in a $75 \mathrm{~g}$ glucose tolerance test. Lactate and pyruvate levels in the CSF were $4.0 \mathrm{mmol} / 1$ (normal: $1.45 \pm$ $0.34 \mathrm{mmol} / \mathrm{l}, \mathrm{n}=20$ ) and $128.4 \mu \mathrm{mol} / \mathrm{l}$ (normal: $105.6 \pm$ $15.9 \mu \mathrm{mol} / 1, \mathrm{n}=20)$, respectively, the former being significantly elevated. Alanine levels in the CSF and plasma were 49 and $455.9 \mathrm{mmol} / \mathrm{l}$, respectively, (normal: $31.9 \pm$
$6.9 \mathrm{mmol} / \mathrm{l}, \mathrm{n}=11$ and $373 \pm 90 \mathrm{mmol} / \mathrm{l}, \mathrm{n}=19)$, being slightly elevated in the CSF. Protein and IgG levels in the CSF were slightly elevated (60 and $9.5 \mathrm{mg} / \mathrm{dl}$, respectively). Folate levels in the CSF and serum were normal at 16.9 and $1.9 \mathrm{ng} / \mathrm{ml}$, respectively (normal: $16.3 \pm 3.3 \mathrm{ng} / \mathrm{ml}, \mathrm{n}=9$ and $4 \cdot 6 \pm 2 \cdot 0 \mathrm{ng} / \mathrm{ml}, \mathrm{n}=34$ ).

Diffuse spike and waves were noted in EEG. Needle electromyography was normal. Motor nerve conduction velocities were $59.2 \mathrm{~m} / \mathrm{s}$ in the right median nerve and $37.5 \mathrm{~m} / \mathrm{s}$ in the right posterior tibial nerve. Somatosensory evoked potentials (SEP) were normal. Low density, and low intensity areas were observed in the bilateral occipital regions by $C T$ and MRI examinations respectively (fig ld). Calcification was noted in the bilateral basal ganglia. Electrocardiography was normal.

\section{Material and methods}

Biopsy was performed in the left quadriceps muscles for cytochemical, immunocytochemical and electron microscopic studies as well as measurement of enzyme activity in mitochondrial electron transport system.

Cytochemistry Skeletal muscle was frozen in acetone dryice. Serial cryostat sections $8 \mu \mathrm{m}$ thick were cut, and stained with $\mathrm{H}$ and $\mathrm{E}$, modified Gomori-trichrome, routine ATPase, NADH, succinate dehydrogenase and cytochrome c oxidase (CCO). CCO activity was evaluated by the method of

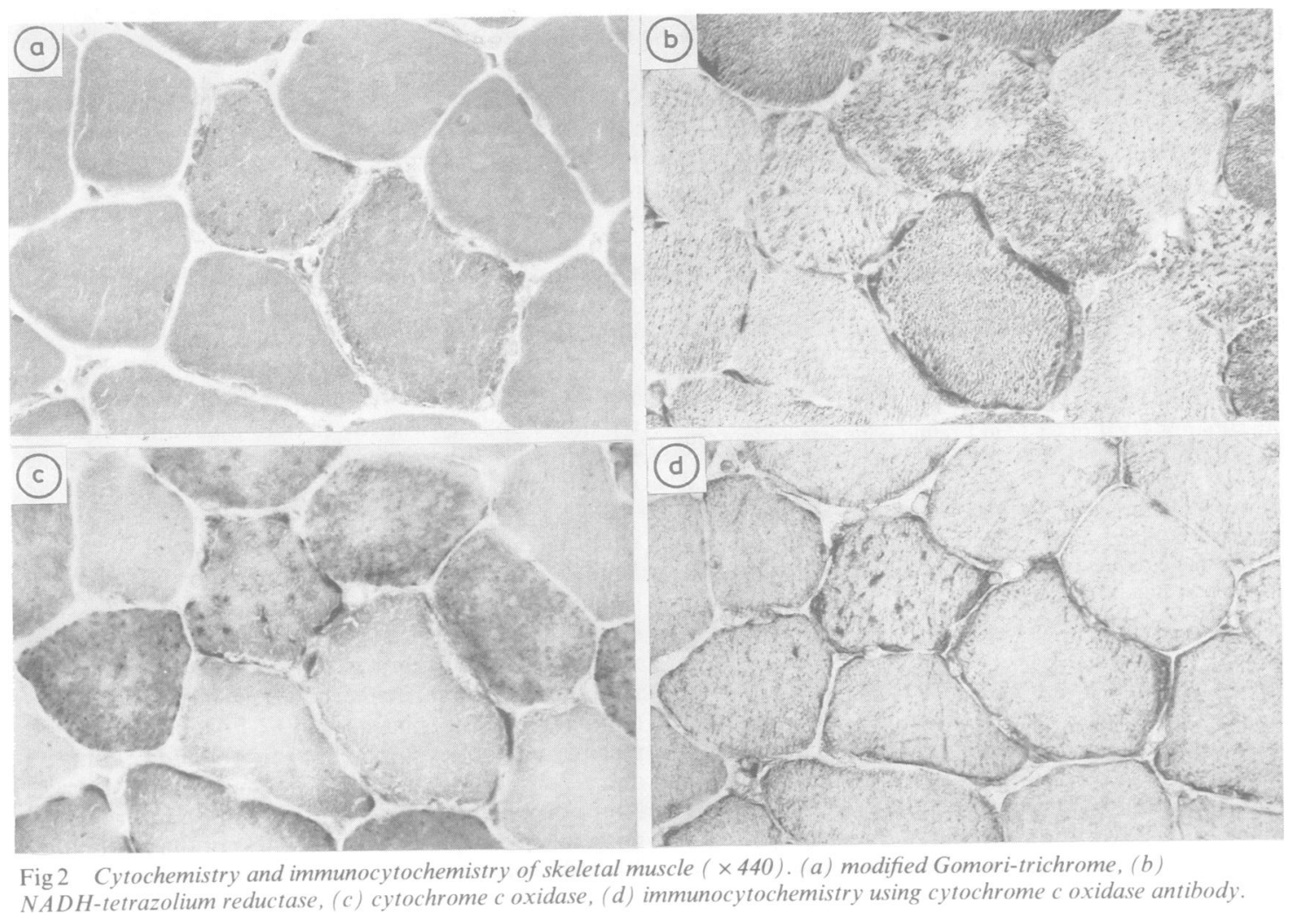




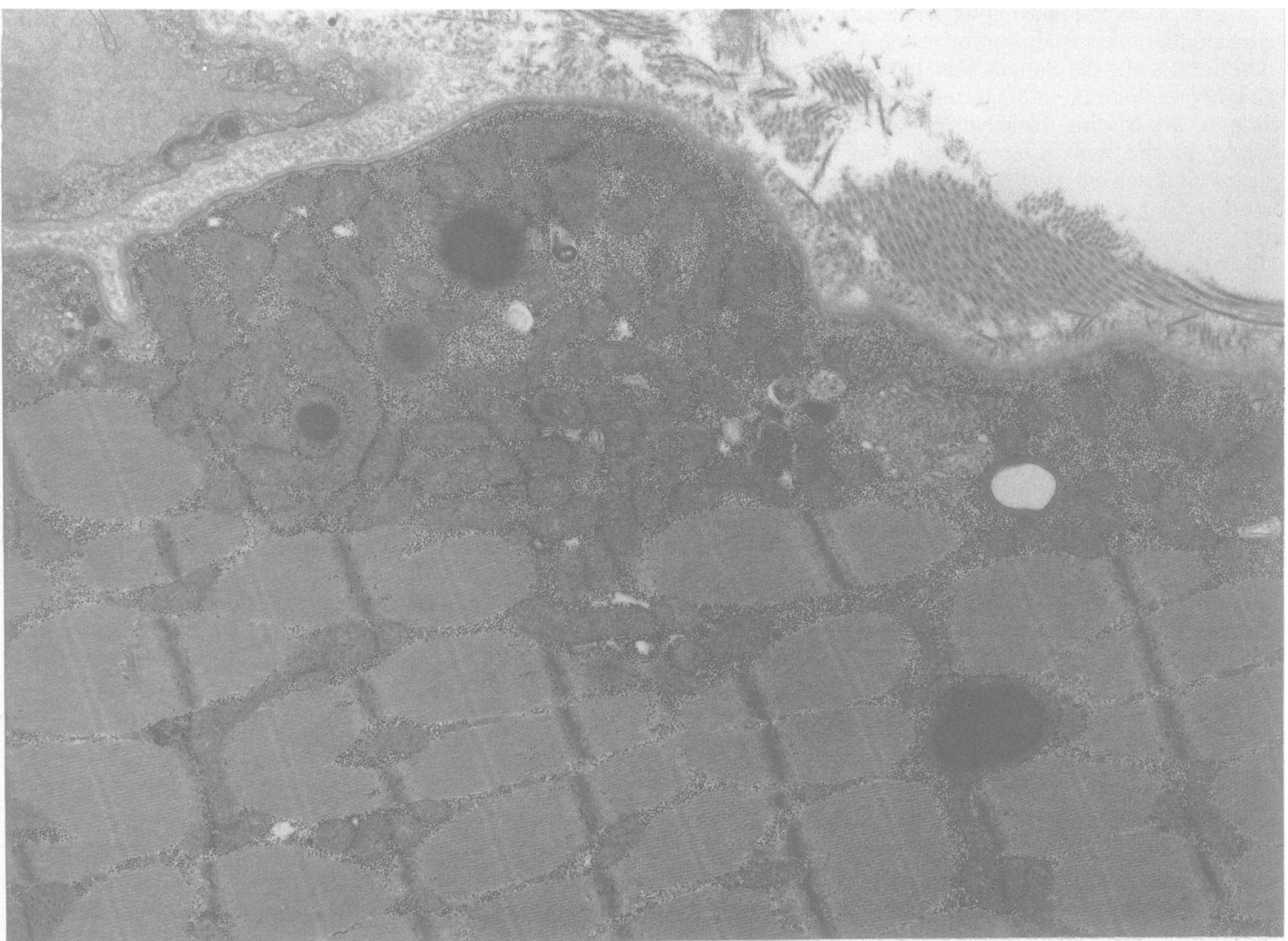

Fig 3 Electromicrography of skeletal muscle, showing abnormal accumulation of mitochondria in the subsarcolemmal space and giant mitochondria containing osmophilic granules in the matrix. $\times 11000$.

Seligmant $e t a^{3}$ with some modification.

Immunocytochemistry To detect enzyme protein of $\mathrm{CCO}$, we performed $\mathrm{CCO}$ antibody stain. Anti-CCO antibody was examined by the avidin-biotin complex method. CCO was purified by the method of Fowler et al, ${ }^{4}$ and electrophoresed with $18 \%$ polyacrylamide plate gel. Rabbits were immunised with subunits I-VII of CCO in Freund complete and incomplete adjuvant.

Enzyme activities in mitochondria Specimens for enzyme assay were stored at $-196^{\circ} \mathrm{C}$ in liquid nitrogen. The enzymes examined were: Cytochrome c oxidase, ${ }^{5}$ succinate cytochrome c reductase and rotenon sensitive NADH cytochrome c reductase, ${ }^{6}$ complex II, $^{7}$ and citrate synthase. ${ }^{8}$

\section{Results}

Ragged-red fibres were observed by modified Gomori-trichrome stain (about 7\%). The cytochemistry of CCO showed scattered nonstained muscle fibres (about $6 \%$ ) although all fibres were heavily stained with CCO antibody (fig 2). Nonstained CCO muscle fibres were found in both type I and II fibres, and also in normally stained fibres with the other stains and without ragged-red fibres (fig 2). Electron microscopy showed abnormal accumulation of mitochondria in the subsarcolemmal space and giant mitochondria containing osmophilic granules in the matrix (fig 3). Rotenone sensitive NADH cytochrome c oxidase activity was lower than that in the controls $(26 \%)$. The other mitochondrial enzyme activities were normal (table 1).

\section{Clinical course and administration of CoQ10}

Mild ileus occurred on the second hospital day but was soon relieved. Although lactate and pyruvate levels gradually decreased after admission, administration of CoQ10 was started on 22 May at $30 \mathrm{mg} /$ day and the dose was increased to $60 \mathrm{mg} /$ day 10 days later. On 19 June, plasma and CSF lactate levels and plasma alanine level increased to 4.9 and $5.1 \mathrm{mmol} / 1$ and $77.2 \mathrm{mmol} / 1$, respectively. On $27 \mathrm{June}$, he was temporarily allowed to go home, but on return to the hospital he complained of weakness of the right hand. CoQ10 was discontinued on the occurrence of ileus on 30 June. In August, ileus was relieved and the 
Table 1 Mitochondrial enzymes activities of skeletal muscle

\begin{tabular}{lcl}
\hline & Control & Patient \\
\hline Cytochrome c oxidase & $331 \pm 116$ & 259 \\
Succinate cytochrome c reductase & $368 \pm 175$ & 374 \\
Rotenone sensitive NADH cytochrome & $1075 \pm 500$ & 281 \\
$\quad$ c reductase & $180 \pm 44$ & 209 \\
Complex II & $419 \pm 135$ & 705 \\
Citrate synthase & & \\
\hline
\end{tabular}

Values show $\mathrm{nmol} / \mathrm{min} / \mathrm{mg}$ mitochondrial protein.

Control values show mean $\pm \operatorname{SD}(n=12)$.

CoQ10 administration $(90 \mathrm{mg} /$ day $)$ was resumed. Lactate and pyruvate levels were slightly decreased in both plasma and CSF in November, 1985, but CSF lactate and alanine levels were increased in May, and November 1986 (table 2). His muscle weakness slightly improved. There has been no exacerbation of clinical symptoms. Prior to $\mathrm{CoQ} 10$ administration, patient and his parents gave informed consent.

\section{Discussion}

Our patient showed repeated episodes of severe headache, vomiting, hemianopsia, neurosensory hearing disturbance, dementia, muscle weakness, muscle atrophy, easy fatigability, myoclonic seizure, and loss of consciousness as well as elevated plasma lactate and pyruvate, and CSF lactate pyruvate, alanine levels. The low density areas in CT scans fluctuated (though not consistent with the vasculature), and EEG showed spike and wave. Muscle biopsy revealed ragged-red fibres. These findings are in close agreement with the features of MELAS reported by Pavlakis et al. ${ }^{2}$

Besides the symptoms described by Pavlakis et al, ${ }^{2}$ episodes of ileus were noted in our patient. These episodes may have resulted from abnormal mitochondrial metabolism in the smooth muscle. Kobayashi et al $l^{9}$ biopsied rectal smooth muscles in patients with mitochondrial encephalomyopathy, and observed mitochondrial abnormalities and obstruction of capillary lumens. Bardosi et al ${ }^{10}$ reported a woman who had chronic malnutrition, extremely low body weight, muscle atrophy and polyneuropathy. At necropsy, intestine capillary muscles showed abnormal mitochondrial accumulation and obstruction of capillary lumens. Recurrent episodes of ileus in our patient may have resulted from abnormal mitochondrial metabolism in the intestine smooth muscle as plasma lactate often increased during the episodes of ileus. Greater attention should be paid to systemic symptoms besides CNS symptoms in examining patients with MELAS.

The mechanism of strokelike episodes in MELAS is obscure. Yamamoto et al ${ }^{11}$ and Gouda et al ${ }^{12}$ reported such episodes together with fluctuation of low density areas in CT scans. These changes in CT scans are considered to be important since they may represent ischaemic changes of the brain vessels. Such a fluctuation in low density areas was noted in our patient at the time of the appearance of hemianopsia. He also presented with frequent episodes of severe headache followed by vomiting prior to strokelike episodes. Attacks of headache were noted also by Hart $e a^{a l^{13}}$ and Holliday et al ${ }^{14}$ in patients with mitochondrial encephalomyopathy. This severe headache may be of importance as a prodrome of strokelike episodes.

Pavlakis et $a^{2}$ studied mitochondrial respiratory chain enzymes in four patients with MELAS, and observed defects of cytochrome c oxidase and cytochrome $\mathrm{c}$ reductase. However, such enzyme abnormalities have been demonstrated in few MELAS patients. In our patient, the activity of NADH cytochrome $\mathrm{c}$ reductase was low compared with normal controls whereas the activity of succinate cytochrome c reductase was normal, although there was a wide range of control values of NADH cytochrome c

Table 2 Lactate, pyruvate and alanine levels in plasma and cerebrospinal fluid

\begin{tabular}{|c|c|c|c|c|c|c|c|c|}
\hline & \multicolumn{6}{|l|}{1985} & \multicolumn{2}{|l|}{1986} \\
\hline & 15 April & 9 May & 19 June & 25 July & $21 \mathrm{Aug}$ & 7 Nov & 27 May & $12 \mathrm{Nov}$ \\
\hline $\begin{array}{l}\text { Plasma: } \\
\text { Lactate }(0.90 \pm 0.50 \mathrm{mmol} / \mathrm{l}, \mathrm{n}=20) \\
\text { Pyruvate }(45.4 \pm 22.7 \mu \mathrm{mol} / \mathrm{l}, \mathrm{n}=20) \\
\text { Alanine }(373 \pm 90 \mathrm{mmol} / \mathrm{l}, \mathrm{n}=19)\end{array}$ & $\begin{array}{r}4 \cdot 2 \\
135 \cdot 2 \\
455 \cdot 9\end{array}$ & $\begin{array}{r}2 \cdot 7 \\
51 \cdot 1 \\
-\end{array}$ & $\begin{array}{r}4.9 \\
112 \cdot 5 \\
756 \cdot 8\end{array}$ & $\begin{array}{r}4 \cdot 1 \\
76 \cdot 1 \\
699 \cdot 3\end{array}$ & $\begin{array}{r}3 \cdot 2 \\
79 \cdot 5 \\
579 \cdot 7\end{array}$ & $\begin{array}{r}2 \cdot 0 \\
82 \cdot 9 \\
558 \cdot 7\end{array}$ & $\begin{array}{r}2 \cdot 5 \\
94 \cdot 3 \\
638 \cdot 1\end{array}$ & $\begin{array}{r}1 \cdot 7 \\
87 \cdot 5 \\
591 \cdot 1\end{array}$ \\
\hline $\begin{array}{l}\text { CSF: } \\
\text { Lactate }(1.45 \pm 0.34 \mathrm{mmol} / \mathrm{l}, \mathrm{n}=20) \\
\text { Pyruvate }(105.6 \pm 15.9 \mu \mathrm{mol} / \mathrm{l}, \mathrm{n}=20) \\
\text { Alanine }(31.9 \pm 6.9 \mathrm{mmol} / \mathrm{l}, \mathrm{n}=11)\end{array}$ & $\begin{array}{r}4 \cdot 0 \\
128 \cdot 4 \\
49 \cdot 0 \\
\text { mild ileus }\end{array}$ & $\begin{array}{l}- \\
- \\
\text { Recovery } \\
\text { from ileus }\end{array}$ & $\begin{array}{l}5 \cdot 1 \\
174 \cdot 9 \\
77 \cdot 2 \\
\text { just before } \\
\text { ileus } \\
\text { CoQ10 } \\
60 \mathrm{mg} / \text { day } \\
\times 3 \text { weeks }\end{array}$ & $\begin{array}{c}3.8 \\
151.0 \\
73.9 \\
\\
\text { Recovery } \\
\text { from ileus } \\
\text { CoQ10(-) }\end{array}$ & $\begin{array}{r}4 \cdot 1 \\
153 \cdot 4 \\
52 \cdot 8\end{array}$ & $\begin{array}{l}\text { CoQ10 } \\
90 \mathrm{mg} / \text { day } \\
\times 2 \text { months }\end{array}$ & $\begin{array}{l}\text { CoQ10 } \\
90 \mathrm{mg} / \text { day } \\
\times 9 \text { months }\end{array}$ & $\begin{array}{l}\text { CoQ10 } \\
90 \mathrm{mg} / \text { day } \\
\times 14 \text { months }\end{array}$ \\
\hline
\end{tabular}


reductase. This indicates that the patient had a mild complex I deficiency in skeletal muscle. CCOnonstained fibres were found in NADH-TR positive stained fibres although $\mathrm{CCO}$ activities in muscle mitochondria were not decreased. CCO non-stained fibres were found in muscles without ragged-red fibres. These findings indicate that $\mathrm{CCO}$ is partially impaired in muscle mitochondria. There have been several reports on $\mathrm{CCO}$ deficiency in mitochondrial myopathy ${ }^{15-17}$ other than MELAS. These reports suggested that deficiency of CCO precedes the development of morphological mitochondrial abnormalities. In our patient, we observed partial $\mathrm{CCO}$ deficiency in MELAS. Although $\mathrm{CCO}$ activity in muscle mitochondria is normal, cytochemical and immunocytochemical approaches are useful to examine partial CCO deficiency. However, it is not clear from these findings whether partial CCO deficiency arises as a secondary phenomenon in mitochondrial degeneration, or primary deficiency.

Reductions in blood and CSF folate ${ }^{18}$ and serum $\mathrm{CoQ} 10^{19}$ have been reported in patients with KearsSayre syndrome, also a mitochondrial encephalomyopathy. Improvements in ECG and blood lactate metabolism following the administration of CoQ10 1920 suggest a relationship between KearsSayre syndrome and CoQ10 metabolism. In our patient, however, lactate or pyruvate levels in the plasma decreased but lactate levels in the CSF did not decrease by treatment with CoQ10; also there was no exacerbation of symptoms during the treatment. His muscle weakness slightly improved. This suggests that oral CoQ10 administration does not affect CNS lactate metabolism. Further studies are needed on the effectiveness of the agent for the treatment of MELAS. CSF and serum folate levels were normal in our patient, suggesting that the metabolism of folate and CoQ10 differs between Kears-Sayre syndrome and MELAS.

Although MELAS may be a distinct clinical syndrome as suggested by Pavlakis et al, ${ }^{2}$ biochemical abnormalities or the mechanism of fluctuation of symptoms observed in this disease are as yet unknown. Since the changes in blood and CSF levels of lactate, pyruvate, and alanine in our patient are considered to reflect changes in respiratory metabolism of mitochondria, comparison between these laboratory results and clinical findings may become important.

We thank Dr A Goishi, Matsuyama Red Cross Hospital for his assistance in CT scan photography (fig la, b).

A part of this study was supported by Grant No.61480203 from the Ministry of Education and No.86-04 from National Center for Nervous, Mental and Muscular Disorders of the Ministry of Health and Welfare, Japan.

\section{References}

1 Shapira Y, Harel S, Russel A. Mitochondrial encephalopathy: a group of neuromuscular disorders with defects in oxidative metabolism. Isr J Med Sci 1977;13:161-4.

2 Pavlakis SG, Phillips PC, Dimauro S, et al. Mitochondrial myopathy, encephalopathy, lactic acidosis, and strokelike episodes: A distinct clinical syndrome. Ann Neurol 1984;16:481-8.

3 Sekuzu I, Orii Y, Okunuki K. Studies on cytochrome c I. Isolation, purification and properties of cytochrome $\mathrm{c}$ from beef heart muscle. $J$ Biochem 1960;48:214-25.

4 Seligman AM, Karnovsky MJ, Wasserkurg HL et al. Non-droplet ultrastructural demonstration of cytochrome oxidase activity with a polymerising osmophilic reagent, diaminobenzidine (DAB). J Cell Biol 1968;38:1-14.

5 Fowler LR, Richardson SH, Hatefi Y. A rapid method for the preparation of highly purified cytochrome oxidase. Biochem Biophys Acta 1962;64:170-3.

6 King TE. Preparations of succinate-cytochrome c reductase and the cytochrome b-c particle, and reconsititution of succinate cytochrome $\mathrm{c}$ reductase. Meth Enzym 1967;10:216-25.

7 Ziegler D, Rieske JS. Preparation and properties of succinate dehydrogenase-coenzyme $Q$ reductase (Complex II). Meth Enzym 1967;10:231-5.

8 Morgan-Hughes JA, Darveniza P, Kahn SN, et al. A mitochondrial myopathy characterized by a deficiency in reduced cytochrome b. Brain 1977;100:617-40.

9 Kobayashi Y, Miyabashi S, Takada S, et al. Ultrastructural study of the childhood mitochondrial myopathic syndrome associated with lactic acidosis. Eur J Pediatr 1982;193:25-30.

10 Bardosi A, Dimauro S, Servidei S, et al. Myo-, neuro-, gastro-, intestino-encephalopathy (MNGIEsyndrome), a new mitochondrial multisystem disorder. Muscle Nerve 1986;9 suppl:177.

11 Yamamoto T, Beppu H, Tsubaki T. Mitochondrial encephalomyopathy: flucuating symptoms and CT. Neurology 1984;34:1456-60.

12 Goda S, Ishimoto S, Ohnishi A, et al. Mitochondrial encephalomyopathy: A case with MELAS (mitochondrial myopathy, encephalopathy, lactic acidosis and strokelike episodes). Clin Neurol (in Japanese) 1985;25:216-26.

13 Hart Z, Chang C, Perrin E, etal. Familal poliodystrophy, mitochondrial myopathy, and lactic acidemia. Arch Neurol 1977;34:180-5.

14 Holliday PL, Climie AR, Gikoy J, et al. Mitochondrial myopathy and encephalopathy; three cases A deficiency of NADH-CoQ dehydrogenase? Neurology 1983;33:1619-21. 
15 Müller-Höcker J, Pongrator D, Deufel TH, et al. Fatal lipid strage myopathy with deficiency of cytochromec-oxidase and carnitine. Virchows Arch Pathol Anat 1983;399:11-23.

16 Johnson MA, Turnbull DM, Dick DJ, et al. A partial deficiency of cytochrome c oxidase in chronic progressive external ophthalmoplegia. $J$ Neurol Sci 1983;60:31-53.

17 Byrne E, Dennett X, Trounce I, et al. Partial cytochrome oxidase (aa3) deficiency in chronic progressive external ophthalmoplegia, histochemical and biochemical studies. J Neurol Sci 1985;71:275-1.

18 Allen RJ, Dimauro S, Coulter DL, et al. Kearns-Sayre syndrome with reduced plasma and cerebrospinal fluid folate. Ann Neurol 1983;13:679-82.

19 Ogasawara S, Yorifugi S, Nishikawa Y, et al. Improvement of abnormal pyruvate metabolism and cardiac conduction defect with coenzyme Q10 in Kearns-Sayre syndrome. Neurology 1985;35:372-7.

20 Ogasawara S, Nishikawa Y, Yorifuji S, et al. Treatment of Kearns-Sayre syndrome with coenzyme Q10. Neurology 1986;36:45-53. 